\title{
Development of a Training Textbook for Countermeasures to "Verbal Abuse, Physical Assault, and Harassment in Pediatric Medical Settings"
}

\author{
Rie Wakimizu' ${ }^{\text {, Hinako Kuwahara }}{ }^{2}$, Yumiko Saito ${ }^{3}$, Makoto Saito ${ }^{4}$ \\ ${ }^{1}$ Department of Child Health and Developmental Nursing, Faculty of Medicine, University of Tsukuba, Ibaraki, Japan \\ ${ }^{2}$ Department of Nursing, Toranomon Hospital, Tokyo, Japan \\ ${ }^{3}$ Doctoral Program in Nursing Science, Graduate School of Comprehensive Human Sciences, \\ University of Tsukuba, Ibaraki, Japan \\ ${ }^{4}$ Department of Child Health, Faculty of Medicine, University of Tsukuba, Ibaraki, Japan \\ Email: riewaki@md.tsukuba.ac.jp
}

How to cite this paper: Wakimizu, R., Kuwahara, H., Saito, Y. and Saito, M. (2022) Development of a Training Textbook for Countermeasures to "Verbal Abuse, Physical Assault, and Harassment in Pediatric Medical Settings". Health, 14, 63-69. https://doi.org/10.4236/health.2022.141006

Received: December 20, 2021

Accepted: January 16, 2022

Published: January 19, 2022

Copyright $\odot 2022$ by author(s) and Scientific Research Publishing Inc. This work is licensed under the Creative Commons Attribution International License (CC BY 4.0).

http://creativecommons.org/licenses/by/4.0/

\begin{abstract}
Background: Recently, abuse, assault, and harassment in pediatric medical settings have become more serious. We extracted typical problems from a nationwide simultaneous survey on abuse in the pediatric medical field and developed a training textbook to simulate or discuss these issues with colleagues. This study aimed to confirm the validity and usability of the contents of this training textbook. Methods: The textbook was mailed to the heads of 51 medical institutions affiliated with the Society of Ambulatory and General Pediatrics of Japan (SAGPJ) and the participants of the SAGPJ online workshop, who were asked to evaluate the textbook using a three-point scale to answer the following questions and provide opinions and impressions about the textbook. Q1. Do you think the whole content and structure of this textbook are useful? Q2. Would you use this textbook as a training textbook for all staff at your facility? A simple tabulation was performed on the evaluation using a three-point scale, while content analysis was performed on the opinions and impressions of the participants. Results: In response to Q1 and Q2, all 63 staff members from 55 facilities answered "3: Yes." Opinions and impressions on the training textbook included "I think it can be used for workplace training" and "it is useful for exchanging various opinions among staff members and gaining a common opinion." Conclusion: We developed a training textbook on abuse, assault, and harassment in pediatric medical settings based on a nationwide survey and confirmed the validity and usability of its contents.
\end{abstract}




\section{Keywords}

Pediatric, Safety Management, Textbook, Validation Study, Workplace Violence

\section{Introduction}

In Japan, a survey of university hospitals [1] reported that $44.3 \%$ of employees had experienced some form of violence in the hospital within the past year, including verbal abuse and violence. Experiences such as abuse and violence from patients and their families have a great impact on the staff's work and may cause them to leave their jobs [2]. However, many experienced staff have adapted to such experiences, and although they feel anxious about their daily work, they continue to work with no one to consult [1] [3]. Conversely, in overseas pediatric medical settings (ward, outpatient department, and emergency department), violence from the patient's family is high [4] [5], and most staff have experienced verbal abuse and physical assault [6] [7] [8].

With the transition from an extended family to a nuclear family in Japan and growing up amid a few siblings and friends due to the decreasing birthrate, the generation of new and upcoming parents lack the experience of interacting with people with diverse values, ways of thinking, behaviors, and cultures. They are unable to realize that society is made up of themselves and others, and lack the imagination to think about the natural distance between them and others, consideration for their surroundings, and how their comments will be perceived [9]. Therefore, there are many families who make unreasonable demands and parents who use abuse or violence as an outlet for accumulated stress [9].

We conducted a national survey on the actual conditions of verbal abuse, physical assault, and harassment from patients and families received by staff within the past year in the pediatric medical setting [10]. Upon analyzing data of 3605 respondents (nurses, clerical workers, physicians, nursery teachers, and nursing assistants) from 521 facilities (79.5\% of which were pediatric clinics and recovery rate of $22.4 \%$ ), the results revealed that $10.5 \%$ of respondents have experienced harm, with verbal abuse being the most common, followed by harassment and physical assault. In terms of measures to manage verbal abuse and violence within the facilities, $10.7 \%$ had manuals and guidelines, while $9.0 \%$ had training programs; however, only $7.3 \%$ of respondents reported that these measures were effective in managing these events, of those who had experienced harm $(45.6 \%)$.

\section{Objective}

We created a training textbook consisting of four typical problems collated and extracted from a nationwide survey [10] on the actual conditions of verbal abuse, physical assault, and harassment from patients and their families experienced by 
staff in the pediatric medical setting. The goal was to improve the staff's ability to respond to each case (problems, necessary measures for prevention, and countermeasures for the event) by examining the cases from the perspective of the "individual" and the "staff." This study aimed to confirm the validity and usability of the contents of this training textbook.

\section{Methods}

\subsection{Training Textbook}

The structure of the training textbook was as follows. The entire content of the 13-page spread color version $4 \mathrm{~A}$ booklet, including the front and back covers, is shown in Document 1 (in supporting information):

1) "Introduction" and Table of Contents: one page each.

2) Summary of the actual damage results revealed in the national survey: Page 2.

3) Four typical problems (each problem is a story consisting of four-frame comics): one page each.

4) Columns for "individual" thoughts and "staff" discussions about "problems (concerns)," "preventive measures (necessary measures for prevention)," and "countermeasures (in the event)": one page each.

5) Column for writing a review of the entire training and realizations: one page.

6) Introduction of four types of recommended in-hospital posters: one page.

\subsection{Targets of the Survey}

The targets of the survey were employees working at medical institutions to which the delegates of the Society of Ambulatory and General Pediatrics of Japan (SAGPJ) belong $(a)$ and pediatric healthcare professionals nationwide who participated in the SAGPJ Annual Meeting Online Workshop $(\mathrm{b})$.

\subsection{Survey Period}

The survey period was between April 2020 (a) and August 2020(b).

\subsection{Data Collection and Survey Content}

We sent the training textbook to the survey participants and asked them to answer the following questions using a three-point scale (3: Yes; 2: Neither; and 1: No) to confirm the validity and usability of the contents:

Q1. Do you think the whole content and structure of this textbook, including the examples, are useful?

Q2. Would you use this textbook as a training textbook for all staff at your facility?

Simultaneously, we requested opinions and impressions on the training textbook. Subjects submitted their response online or via the post. Each subject was asked to write the name of the institution to which they belong in addition to Q1 
and Q2, but anonymous responses were allowed.

\subsection{Data Analysis}

For the analysis, a simple tabulation was performed on the evaluation using a three-point scale, while content analysis was performed on the opinions and impressions of the participants.

\subsection{Ethical Consideration}

This study was conducted with the approval of the Medical Ethics Review Board (approval number: 1238) at the University of Tsukuba.

\section{Results}

Of the 51 facilities to which the SAGPJ delegates belong, 54 managers from 46 facilities and nine employees ( 2 doctors, 7 medical staff) from nine facilities who joined the SAGPJ online workshop participated in this survey. In response to Q1 and Q2 above, all 63 staff members from 55 facilities answered "3: Yes" (Table 1 and Table 2). Table 3 shows the comments, opinions, and impressions from the subjects regarding the validity and usability of the content of the training textbook (Document 1).

\section{Document 1}

https://shouni-kazoku.jp/update/wp-content/uploads/2021/02/kensyu.pdf.

\section{Discussion}

The results of the survey confirmed the validity and usability of the content of the training text we developed. The evaluation of the text was uniformly high, and many commended the content of the text even when referring to the comments written in the free comment text box. A participant even ordered 30

Table 1. Question 1: Do you think the whole content and structure of this textbook, including the examples, are useful?

\begin{tabular}{cc}
\hline & $(\mathrm{N}=63)$ \\
\hline Yes & 63 \\
Neither & 0 \\
No & 0 \\
\hline
\end{tabular}

Table 2. Question 2: Would you use this textbook as a training textbook for all staff at your facility?

\begin{tabular}{cc}
\hline & $(\mathrm{N}=63)$ \\
\hline Yes & 63 \\
Neither & 0 \\
No & 0 \\
\hline
\end{tabular}


Table 3. Comments, opinions, and impressions on the validity and usability of the textbook content.

\begin{tabular}{|c|c|}
\hline \multicolumn{2}{|l|}{$(\mathrm{N}=63)$} \\
\hline Contents on the opinions and impressions & $\begin{array}{l}\text { Number of } \\
\text { mentions }\end{array}$ \\
\hline $\begin{array}{l}\text { It can be used in workplace training and for exchanging various } \\
\text { opinions among staff members to form a common opinion. }\end{array}$ & 33 \\
\hline $\begin{array}{l}\text { Since the cases are often encountered in the field, it is a good } \\
\text { material for sharing and discussing our own experiences. }\end{array}$ & 23 \\
\hline $\begin{array}{l}\text { I find it greatly useful as a textbook for workshops and trainings } \\
\text { not only in my workplace but also with people who work in } \\
\text { other workplaces. }\end{array}$ & 21 \\
\hline $\begin{array}{l}\text { The case consists of four-frame comics, and it is easy to } \\
\text { understand the position of the story line and characters }\end{array}$ & 10 \\
\hline $\begin{array}{l}\text { Since it is a thin textbook, we were able to use it to confirm the } \\
\text { conditions at our respective workplaces (what can be applied at our } \\
\text { hospital and what our future tasks are) through discussions } \\
\text { between the staff during breaks. }\end{array}$ & 8 \\
\hline It could be used as a training textbook for medical safety. & 5 \\
\hline $\begin{array}{l}\text { We used the textbook in discussions among staff and how we } \\
\text { could deal with the patient's family to prevent problems. }\end{array}$ & 2 \\
\hline $\begin{array}{l}\text { When we tried role-playing among the staff for each case } \\
\text { presented in the textbook, we realized the necessity to talk to } \\
\text { the patient's family and among the staff more than before. }\end{array}$ & 1 \\
\hline $\begin{array}{l}\text { I believe it would be better if there were suggestions as to the best } \\
\text { way to handle the patient's family in accordance with the law. }\end{array}$ & 1 \\
\hline
\end{tabular}

training textbooks (shipped free of charge) stating, "I want to share the contents of this textbook with all the staff at my hospital and have an opportunity for a discussion."

Using this training textbook, we will continue to implement online intervention programs to strengthen 1) the ability of the individual staff to respond to such situations, 2) administrative measures, and 3) team measures against verbal abuse, physical assault, and harassment. Employees who are concerned about complaints from patients and their families will be informed of the viewpoint/awareness and actual measures necessary for safe response and prevention, while facility managers will be provided with knowledge of measures necessary to protect employees. Intervention effects by reporting tools [11], education [12] [13] [14], and projects [15] have also been reported in overseas studies. We believe that it is important to create a comfortable and safe working environment by strengthening employees' ability to respond to patients and families while equipping them with a common perspective and awareness. 


\section{Conclusion}

We examined the validity and usability of the content of the training textbook on the steps to take in case of abuse of healthcare workers by patients and caregivers in pediatric medical settings that developed based on a nationwide survey in Japan. We found that the content of the textbook was highly usable as a training textbook for employees working at pediatric medical institutions throughout Japan.

\section{Acknowledgements}

We would like to thank Editage (https://www.editage.com) for English language editing.

\section{Funding}

This research was funded by the Grant-in-Aid for Challenging Exploratory Research from the Ministry of Education, Culture, Sports, Science, and Technology; Japan (18K19664) (principal investigator: Rie Wakimizu).

\section{Conflicts of Interest}

The authors declare no conflicts of interest regarding the publication of this paper.

\section{References}

[1] Iwao, A., Fujiwara, K., Hasegawa, S., Ueno, K., Oota, H., Hasegawa, S., Sakurai, J., Aida, H., Nakazawa, K., Koichi, K., Furuhata, H., Nakano, Y., Kaneko, E., Inagaki, H., Yanagi, T., Kitahara, R., Yamashita, S. and Ochiai, K. (2013) The Actual State of In-Hospital Violence from Patients and Their Families against the Staff of the Main Hospitals of Private Universities in Tokyo: Collaborative Study by the Private University Hospital Liaison Council for Medical Safety Promotion. Journal of the Japan Society for Healthcare Administration, 50, 219-227.

[2] Kaneko, T., Ishibashi, M. and Hibi, M. (2009) Patient Harassment of Medical Staff in Japan. The Journal of Japan Society for Health Care Management, 10, 399-403.

[3] Gerberich, S.G., Church, T.R., McGovern, P.M., Hansen, H.E., Nachreiner, N.M., Geisser, M.S., Ryan, A.D., Mongin, S.J. and Watt, G.D. (2004) An Epidemiological Study of the Magnitude and Consequences of Work-Related Violence: The Minnesota Nurses' Study. Occupational and Environmental Medicine, 61, 495-503. https://doi.org/10.1136/oem.2003.007294

[4] Shaw, J. (2015) Staff Perceptions of Workplace Violence in a Pediatric Emergency Department. Work, 51, 39-49. https://doi.org/10.3233/WOR-141895

[5] Li, Z., Yan, C.M., Shi, L., Mu, H.T., Li, X., Li, A.Q., Zhao, C.S., Sun, T., Gao, L., Fan, L.H. and $\mathrm{Mu}, \mathrm{Y}$. (2017) Workplace Violence against Medical Staff of Chinese Children's Hospitals: A Cross-Sectional Study. PLoS ONE, 12, e0179373. https://doi.org/10.1371/journal.pone.0179373

[6] Derscheid, D.J. and Arnetz, J.E. (2020) Patient and Family Member Violent Situations in a Pediatric Hospital: A Descriptive Study. Journal of Pediatric Nursing, 55, 241-249. https://doi.org/10.1016/j.pedn.2020.07.014 
[7] McAneney, C.M. and Shaw, K.N. (1994) Violence in the Pediatric Emergency Department. Annals of Emergency Medicine, 23, 1248-1251.

https://doi.org/10.1016/S0196-0644(94)70349-3

[8] Partridge, B. and Affleck, J. (2017) Verbal Abuse and Physical Assault in the Emergency Department: Rates of Violence, Perceptions of Safety, and Attitudes towards Security. Australasian Emergency Nursing Journal, 20, 139-145.

https://doi.org/10.1016/j.aenj.2017.05.001

[9] Nagai, H. (2009) Sociology of Helicopter Parent. Journal of the Faculty of Contemporary Society, Toyama University of International Studies, 1, 123-131.

[10] Wakimizu, R., Miki, A., Saito, M., Ohto, T., Fukushima, T., Imai, H., Maeno, T., Kuwahara, H., Koyama, S. and Kuroki, H. (2019) Current State and Management of Verbal Abuse, Violence, Sexual Harassment and Other Harassment by Families in Pediatric Medical Settings. The Journal of Ambulatory and General Pediatrics, 22, 452-461.

[11] Stene, J., Larson, E., Levy, M. and Dohlman, M. (2015) Workplace Violence in the Emergency Department: Giving Staff the Tools and Support to Report. The Permanente Journal, 19, e113-117. https://doi.org/10.7812/TPP/14-187

[12] Koller, L.H. (2016) It Could Never Happen Here: Promoting Violence Prevention Education for Emergency Department Nurses. The Journal of Continuing Education in Nursing, 47, 356-360. https://doi.org/10.3928/00220124-20160715-06

[13] Wong, A.H., Wing, L., Weiss, B. and Gang, M. (2015) Coordinating a Team Response to Behavioral Emergencies in the Emergency Department: A Simulation-Enhanced Interprofessional Curriculum. Western Journal of Emergency Medicine, 16, 859865. https://doi.org/10.5811/westjem.2015.8.26220

[14] Ming, J.L., Tseng, L.H., Huang, H.M., Hong, S.P., Chang, C.I. and Tung, C.Y. (2019) Clinical Simulation Teaching Program to Promote the Effectiveness of Nurses in Coping with Workplace Violence. Chinese Journal of Nursing, 66, 59-71.

[15] Schwartz, F. and Bjorklund, P. (2019) Quality Improvement Project to Manage Workplace Violence in Hospitals: Lessons Learned. Journal of Nursing Care Quality, 34, 114-120. https://doi.org/10.1097/NCQ.0000000000000358 